\title{
Genetic association of polymorphism rs2230054 in $C X C R 2$ gene with gout in Chinese Han male population
}

\author{
YANGANG WANG ${ }^{1}$ YANQUN WU ${ }^{2}$, QIAN XING ${ }^{2}, N A N C H U^{3}$, LIYAN SHEN ${ }^{1}$, XINJUAN YU ${ }^{4}$, \\ LILI WANG \\ ${ }^{1}$ Department of Endocrinology, The Affiliated Hospital of Qingdao University, Qingdao, Shandong China \\ ${ }^{2}$ Department of Immune Rheumatology, Qingdao Municipal Hospital, Qingdao, Shandong, China \\ ${ }^{3}$ Department of Endocrinology, Qingdao Hiser Medical Group, Qingdao, Shandong, China \\ ${ }^{4}$ Department of Central Lab, Qingdao Municipal Hospital, Qingdao, Shandong, China
}

\begin{abstract}
Neutrophils are crucial in the process of gout flare and remission. The signal transduction pathway of chemokine plays a vital role in the chemotaxis and activation of neutrophils. CXCR2 gene knocked out can avoid the acute neutrophilic inflammation stimulated by monosodium urate (MSU) crystals in mice. To investigate the relationship among CXCR1 rs2234671, CXCR2 rs1126579, and rs2230054 polymorphisms with gout arthritis flare in the Chinese Han male population, a case-control study was carried out in 412 gout patients and 508 gout-free individuals. TaqMan probes fluorescence real-time polymerase chain reaction (PCR) was used to genotype CXCR1 rs2234671, CXCR2 rs1 126579, and rs2230054 SNPs. There was a clear link between CXCR2 rs2230054 T included genotypic and T allelic frequencies and gout cases ( $c^{2}=9.286, p=0.002$ by genotype, $c^{2}=8.639, p=0.003$ by allele), while no significant differences were observed between the gouty arthritis group and the control group in CXCR1 rs2234671 and CXCR2 rs1126579 genotypic and allelic frequencies. Multivariate logistic regression analysis showed that the T genotype included in rs2230054 can decrease the risk of gouty arthritis (adjusted OR $=0.47 ; 95 \%$ CI: 0.31-0.74) compared with the CC genotype. Our study might suggest that rs2230054 in CXCR2 is associated with susceptibility to gout in Chinese males.
\end{abstract}

Key words: CXCR1, CXCR2, gout, polymorphism, Chinese.

(Centr Eur J Immunol 2020; 45 (1): 80-85)

\section{Introduction}

Gout is a familiar non-infected auto-inflammatory arthritis, with elevated serum uric acid level and precipitation of monosodium urate (MSU) crystals in the joints and around tissues. About $0.9-2 \%$ of adults are suffering from this disease in China and developed countries [1-4]. Although elevated urate demonstrates significant correlation with gout $[5,6]$, only about $10 \%$ of hyperuricemia patients develop into clinical gout, suggesting that hyperuricemia alone cannot account for the occurrence of gout arthritis [7]. More and more evidences has indicated that environmental factors, genetic susceptibility, and immune disorders may be responsible for the development of gout arthritis [8]. Previous genetic researches including genome-wide association studies (GWASs) have identified dozens of loci related with gout flare, in which interleukin (IL)-1 $\beta$, IL-6, and IL-8 (CXCL8) are involved [9-13].
As the precipitation of MSU crystals in the joint could induce gout flare, which is proved in cell lines and mouse models studies, it is widely recognized that MSU crystals are the endogenous danger signal of the innate immune system components $[14,15]$, characterized by the recruitment of neutrophils into the joints. It is thought that $\mathrm{T}$ cells regulate its pathogenesis through proinflammatory cytokines such as IL- $1 \beta$, tumor necrosis factor (TNF)- $\alpha$, CXCL8, and IL-6 [16, 17].

Normally, neutrophils are scarce in synovial fluid, therefore, the enhancement of neutrophil-endothelial adhesion is necessary in acute gout arthritis (GA) onset. At the same time, neutrophil transmigration induced by newly generated neutrophil chemotaxins is similarly necessary in acute gout. The chemotactic factors $(\mathrm{CCF})$ induced by the crystal are assigned to neutrophil chemotactic $\mathrm{C}-\mathrm{X}-\mathrm{C}$ chemokines, in which CXCL8 is involved [18-20]. CXCL8 is rich in the synovia in acute GA, and is responsible for

Correspondence: Yangang Wang, Department of Endocrinology, The Affiliated Hospital of Qingdao University, 16 Jiangsu Rd, 266000 Qingdao, China, e-mail: wangyang1966@126.com Submitted: 12.07.2017; Accepted: 18.09.2017 
more than $90 \%$ of the neutrophil chemotactic activity that gathers in the MSU crystals, stimulated site in vitro [21].

$\mathrm{C}-\mathrm{X}-\mathrm{C}$ receptor $(C X C R)-1$ and $C X C R 2$ are specific receptors for the chemokine interleukin 8 (IL-8, CXCL8). Cellular activities of CXCL8 are mediated by $C X C R 1$ and $C X C R 2$ (IL-8R $\beta$ ), which maintain $78 \%$ of amino acid similarity and are encoded by two single-copy genes that are located on chromosome 2q34-35 [22]. Earlier studies on $C X C R$ in gout have been mainly concentrated on animal models about arthritis induced by MSU crystals. Santos et al. demonstrated that that IL-1 $\beta$ drives CXCL1 and consequent $C X C R 2$-dependent recruitment of neutrophils [23]. The inhibition of either receptor was able to reduce the influx of neutrophils and associated pain induced by MSU crystals [23]. The functional homologue of CXCR2 was located in the chromosome $1 \mathrm{C} 3$ in rats and mice, while $C X C R 1$ was absent in them. It was proved that $C X C R 2$ homologue gene knock-out mice can avoid the acute neutrophilic inflammation stimulated by urate crystals [24]. The function or expression of many inflammatory cytokines may be influenced by the polymorphisms in their gene loci, which may facilitate the susceptibility or severity of inflammatory diseases. Therefore, we hypothesize that the polymorphisms of $C X C R 1$ or $C X C R 2$ may be associated with GA. This study was the first to establish an association between GA susceptibility and polymorphisms of CXCRI and $C X C R 2$ in a Chinese Han male population. Follow-up functional studies will be needed to validate these findings.

\section{Material and methods}

\section{Subjects}

A total of 412 male gout patients, who visited the Department of Gout at the Affiliated Hospital of Qingdao University and the Department of Rheumatology at the Qing Dao Municipal Hospital between December 2013 and June 2015, were recruited. The patients were diagnosed with gout according to the classification criteria established by the American College of Rheumatology (ACR) (1977) [25]. Patients having a medical history of nephropathy, cancer, hematopathy, autoimmune diseases, other arthritis diseases, or present-day infection were excluded. As the controls, 508 age-matched normal male controls who took regular physical examination at the Affiliated Hospital of Qingdao University between December 2013 and June 2015, with no history of arthritis diseases or systemic inflammatory disease were enrolled in this study at the same time. This study was conducted according to the principles of the Declaration of Helsinki, approved by the Affiliated Hospital, Qingdao University ethical committees, and an informed consent was obtained from all participants.

\section{Examination of regulatory parameters}

Serum uric acid (sUA), triglycerides (TG), total cholesterol (TC), alanine transaminase (ALT), aspartate trans- aminase (AST), serum glucose (GLU), and serum creatinine (Scr) were measured by Hitachi model 7600 series automatic analyzer. All measurements were completed by an expert who was blinded to the study in the Clinical Laboratory Department, the Affiliated Hospital of Qingdao University.

\section{DNA isolation and genetic analyses}

Genomic DNA of the total subjects was isolated from $200 \mu \mathrm{l}$ whole peripheral blood samples using the TIANamp blood DNA kit (Tiangen Biotech, Beijing, Co., Ltd.). TaqMan probes fluorescence real-time polymerase chain reaction (PCR) were used to detect the polymorphisms of rs2234671, rs1126579, and rs2230054 located in CXCR1 and $C X C R 2$, respectively. Biosystems By Life Technologies (New York, USA) was applied to design and synthesize the primers and TaqMan probes. For rs2234671, the forward primer sequence was 5'-ATGAGGACCCAGGTGATCCAGGAGA-3' and the reverse was 5'-CTGTGAGCGCCGCAACAACATCGGC-3'. For rs1126579, the forward primer sequence was 5'-CAGCCCCCATTGTGGTCACAGGAAG-3' and the reverse was 5'-AGAGGAGGCCACGTTCTTACTAGTT-3'. For rs2230054, the forward primer sequence was 5'-TTGCTGTCGTCCTCATCTTCCTGCT-3' and the reverse was 5'-TGCTGGCTGCCCTACAACCTGGTCC-3'. PCR was carried out in a final reaction volume of $25 \mu \mathrm{l}$ containing: PCR master $\operatorname{mix}(2 \times) 12.5 \mu \mathrm{l}$, SNP genotyping assay $(20 \times) 1.25 \mu \mathrm{l}$, DNA sample, and DNase-free water $11.25 \mu$. The amplifications were finished with the conditions as follows: initial denaturation at $95^{\circ} \mathrm{C}$ for 3 minutes, followed denaturation by 45 cycles at $95^{\circ} \mathrm{C}$ for 15 seconds and $60^{\circ} \mathrm{C}$ for 1 minute. During PCR thermal cycle, the fluorescence was detected in real-time. The amplification products were confirmed by sequencing. The genotype discrimination was finally analyzed by Bio-Rad CFX manager 3.0 software.

\section{Statistical analysis}

We used SPSS 18.0 to analyze all the results. The Hardy-Weinberg equilibrium (HWE) test in genotype distribution was carried out by using the goodness-of-fit $\chi^{2}$ test. The Student's $t$-test was employed to compare the differences in demographic and clinical parameters between two groups. Discrimination in the allelic and genotypic frequencies between cases and controls were assessed using the $\chi^{2}$ test with $2 \times 2$ contingency. Relationships in the genotypes distribution and the allelic frequencies between these two groups were measured by calculating the odds ratios (OR) and $95 \%$ confidence intervals (CI) from multivariate logistic regression analysis, with the adjustment for hypertension, diabetes (DM), coronary heart disease (CHD), diastolic blood pressure (DBP), systolic pressure (SBP), waist-hip ratio (WHR), body mass index (BMI), GLU, TG, TC, and 
age, which were possible confounders. The $p$-values $<0.05$ was considered as statistical significance.

\section{Results}

\section{Clinical and laboratory characteristics of all the participants studied}

The clinical and laboratory characteristics of all the participants of this study are summarized in Table 1 . There were no differences between GA cases and controls regarding age and gender. GA patients had significantly higher morbidity in DM, CHD, and hypertension than controls. BMI and WHR in GA patients were also much higher than that in the controls. GA patients were shown to have significantly higher sUA, GLU, TG, TC, and ALT, and lower Scr, SBP, and DBP. As for other parameters such as alcohol consumption and smoking, no significant differences were observed between GA patients' group and controls' group.

\section{Genetic analysis}

Genotypic distributions and allelic frequencies of rs2234671, rs1126579, and rs2230054 in GA patients and controls are shown in Table 2. The genetic distributions of the three SNPs in controls were in HWE. No significant differences were observed between cases and controls in rs2234671 and rs1126579 genotypic and allelic frequencies. CXCR2 rs2230054 T included genotypic and T allelic frequencies in gout cases were different from controls significantly ( $p=0.002$ by genotype, $p=0.003$ by allele). Multivariate logistic regression analysis also suggested that the CC genotype in rs2234671 and rs1126579 do not increase the risk of GA compared with the GG and TT genotype, respectively, while the $\mathrm{T}$ genotype included in rs2230054 can decrease the risk of gouty arthritis compared with the CC genotype.

\section{Discussion}

To our knowledge, this is the first study to reveal the relationship between CXCR SNPs and patients with primary GA in Chinese Han population, which potentially broaden the scope of diseases contributed to CXCR-1 and CXCR-2 SNPs. The direct association of rs2234671 and rs1126579 with gout did not reach significance, which makes us come to the conclusion that these two SNPs may not be genetic risk factors for GA in the studied Chinese Han population. However, the frequencies of the $\mathrm{T}$ allele

Table 1. Characteristics of gout cases and control study samples (mean $\pm \mathrm{SD}$ )

\begin{tabular}{|c|c|c|c|c|}
\hline Parameter & GA $(n=412)$ & Control $(n=508)$ & $t$ or $\chi^{2}$ & $p$-value \\
\hline Age (years) & $46.04 \pm 13.61$ & $46.65 \pm 10.04$ & 0.761 & 0.447 \\
\hline Gender (male/female) & $412 / 0$ & $508 / 0$ & - & - \\
\hline Alcohol consumption, $n(\%)$ & $200(48.5)$ & $262(51.6)$ & 0.836 & 0.361 \\
\hline Smoking, $n(\%)$ & $150(36.4)$ & $209(41.1)$ & 2.143 & 0.143 \\
\hline $\mathrm{DM}, n(\%) \#$ & $38(9.2)$ & $22(4.3)$ & 8.933 & 0.003 \\
\hline CHD, $n(\%)$ & $28(6.8)$ & $4(0.8)$ & 24.465 & 0.000 \\
\hline Hypertension, $n(\%)^{*}$ & $134(32.5)$ & $72(14.2)$ & 44.086 & 0.000 \\
\hline $\mathrm{BMI}\left(\mathrm{kg} / \mathrm{m}^{2}\right)$ & $27.43 \pm 3.56$ & $25.30 \pm 3.57$ & 9.038 & 0.000 \\
\hline WHR & $0.93 \pm 0.07$ & $0.90 \pm 0.06$ & 7.199 & 0.000 \\
\hline $\mathrm{SBP}(\mathrm{mm} \mathrm{Hg})$ & $129.53 \pm 15.82$ & $135.60 \pm 20.58$ & 5.056 & 0.000 \\
\hline $\mathrm{DBP}(\mathrm{mm} \mathrm{Hg})$ & $82.61 \pm 23.18$ & $86.47 \pm 11.39$ & 3.331 & 0.001 \\
\hline $\mathrm{sUA}(\mu \mathrm{mol} / \mathrm{l})$ & $514.89 \pm 85.26$ & $352.85 \pm 70.17$ & 30.991 & 0.000 \\
\hline $\operatorname{Scr}(\mu \mathrm{mol} / \mathrm{l})$ & $82.61 \pm 23.18$ & $86.47 \pm 11.39$ & 3.096 & 0.002 \\
\hline GLU (mmol/l) & $6.08 \pm 2.49$ & $5.43 \pm 1.44$ & 4.955 & 0.000 \\
\hline $\mathrm{TG}(\mathrm{mmol} / \mathrm{l})$ & $2.51 \pm 1.32$ & $1.56 \pm 1.31$ & 10.848 & 0.000 \\
\hline $\mathrm{TC}(\mathrm{mmol} / \mathrm{l})$ & $5.43 \pm 1.11$ & $5.28 \pm 0.99$ & 2.119 & 0.034 \\
\hline $\operatorname{ALT}(\mathrm{U} / \mathrm{l})$ & $34.52 \pm 23.54$ & $27.52 \pm 17.60$ & 5.002 & 0.000 \\
\hline AST (U/l) & $23.39 \pm 11.83$ & $23.03 \pm 11.08$ & 0.474 & 0.636 \\
\hline
\end{tabular}

"fasting plasma glucose (FPG) $\geq 7.8$ mmol/l or oral glucose tolerance test (OGTT) 2 h plasma glucose $\geq 11.1$ mmol/l or ongoing antidiabetics therapy, ${ }^{*}$ blood pressure $\geq 140 / 90 \mathrm{~mm} \mathrm{Hg}$ or ongoing antihypertensive therapy, GA - gouty arthritis, DM - diabetes, CHD - coronary heart disease, BMI - body mass index, WHR - waist-hip ratio, SBP - systolic pressure, DBP - diastolic blood pressure, sUA - serum uric acid, Scr-serum creatinine, GLU - serum glucose, TG - triglycerides, $T C$ - total cholesterol, ALT - alanine transaminase, AST - aspartate transaminase 
Table 2. Allele and genotype frequencies of $C X C R 1$ and $C X C R 2$ SNPs in patients with gout arthritis and controls

\begin{tabular}{|c|c|c|c|c|c|c|}
\hline Allele/genotype & $\begin{array}{c}\text { GA } \\
(n=412) \\
n(\%) \\
\end{array}$ & $\begin{array}{c}\text { Control } \\
(n=508) \\
n(\%)\end{array}$ & $\chi^{2}$ & $p$-value & OR $(95 \%$ CI $)$ & Adjusted OR (95\% CI) \\
\hline \multicolumn{7}{|l|}{ rs2234671 } \\
\hline $\mathrm{GG}^{\text {Reference }}$ & $332(80.6)$ & $409(80.5)$ & - & - & 1.0 & 1.0 \\
\hline GC & 77 (18.7) & $95(18.7)$ & 0.000 & 0.993 & $1.00(0.72-1.39)$ & $1.06(0.72-1.57)$ \\
\hline $\mathrm{CC}$ & $3(0.7)$ & $4(0.8)$ & 0.000 & 1.000 & $0.92(0.21-4.16)$ & $2.09(0.37-11.69)$ \\
\hline HWE $^{*}$ & $\begin{array}{l}\chi^{2}=0.412 \\
p=0.814\end{array}$ & $\begin{array}{l}\chi^{2}=0.354 \\
p=0.838\end{array}$ & & & & \\
\hline $\mathrm{G}^{\text {Reference }}$ & $741(89.9)$ & $913(89.9)$ & - & - & 1.0 & \\
\hline $\mathrm{C}$ & $83(10.1)$ & $103(10.1)$ & 0.002 & 0.963 & $0.99(0.73-1.35)$ & \\
\hline \multicolumn{7}{|l|}{ rs1126579 } \\
\hline $\mathrm{TT}^{\text {Reference }}$ & $133(32.3)$ & $190(37.4)$ & - & - & 1.0 & 1.0 \\
\hline $\mathrm{TC}$ & $201(48.8)$ & $235(46.3)$ & 1.826 & 0.177 & $1.22(0.91-1.63)$ & $1.33(0.94-1.87)$ \\
\hline $\mathrm{CC}$ & $78(18.9)$ & $83(16.3)$ & 2.310 & 0.129 & $1.34(0.92-1.96)$ & $1.41(0.89-2.21)$ \\
\hline $\mathrm{HWE}^{*}$ & $\begin{array}{l}\chi^{2}=0.018 \\
p=0.991\end{array}$ & $\begin{array}{l}\chi^{2}=0.515 \\
p=0.773\end{array}$ & & & & \\
\hline $\mathrm{T}^{\text {Reference }}$ & $467(56.7)$ & $615(60.5)$ & - & - & 1.0 & \\
\hline $\mathrm{C}$ & $357(43.3)$ & $401(39.5)$ & 2.794 & 0.095 & $1.17(0.97-1.41)$ & \\
\hline \multicolumn{7}{|l|}{ rs2230054 } \\
\hline $\mathrm{CC}^{\text {Reference }}$ & $361(87.6)$ & $407(80.1)$ & - & - & 1.0 & 1.0 \\
\hline $\mathrm{CT}$ & $50(12.1)$ & 99 (19.5) & 9.125 & 0.003 & $0.57(0.39-0.82)$ & $0.50(0.32-0.77)$ \\
\hline $\mathrm{TT}$ & $1(0.3)$ & $2(0.4)$ & 0.000 & 1.000 & $0.56(0.05-6.24)$ & $0.02(0.001-0.52)$ \\
\hline HWE & $\begin{aligned} \chi^{2} & =0.285 \\
p & =0.867\end{aligned}$ & $\begin{array}{l}\chi^{2}=0.461 \\
p=0.292\end{array}$ & & & & \\
\hline $\mathrm{CC}^{\text {Reference }}$ & 361 (87.6) & $407(80.1)$ & & & & \\
\hline $\mathrm{CT}+\mathrm{TT}$ & $51(12.4)$ & $101(19.9)$ & 9.286 & 0.002 & $0.57(0.40-0.82)$ & $0.47(0.31-0.74)$ \\
\hline $\mathrm{C}^{\text {Reference }}$ & 772 (93.7) & 913 (89.9) & - & - & 1.0 & \\
\hline $\mathrm{T}$ & $52(6.3)$ & $103(10.1)$ & 8.639 & 0.003 & $0.60(0.42-0.85)$ & \\
\hline
\end{tabular}

${ }^{*}$ HWE-Hardy-Weinberg equilibrium

of $C X C R 2$ rs2230054 was lower in the studied cases than in the controls, which make us believe that the $\mathrm{T}$ allele of $C X C R 2$ rs2230054 may be a protection factor for the development of GA in the studied Chinese Han male population.

The rs2230054 site is a C/T single-nucleotide variation on human chromosome 2 , which polymorphism present on CXCR2 gene Ex3+811C $>$ T causes an amino acid substitution (L262L) in the CXCR-2 protein. As a common receptor of neutrophil chemotaxis, CXCR2 rs2230054 polymorphisms was examined in total joint arthroplasty (TJA) patients with prosthetic joint infection (PJI), and no association was found [26]. However, Hsing et al. [27] verified that rs2230054 genetic variety was associated with the risk of bile duct cancer and biliary stones, when evaluated the role of inflammation-related genes in the development of biliary tract cancer and gallstones. In the present study, we found that $\mathrm{T}$ allele of $C X C R 2$ rs2230054 was related with GA and may be a protection factor for the development of gout. This finding supported the hypothesis that common gene polymorphism in $C X C R 2$ contribute to the etiology of gout in all mankind.

The etiology of gout is complex and genetic risk factors are involved together with the exposure. As previously described, neutrophils are crucial in the process of gout flare and remission, which are among the first cells to arrive at the inflammation site through chemotaxis coming after senses of danger-associated molecular patterns discharged by injured cells. Chemokine signaling pathway plays an important role in regulating neutrophils trafficking by facilitating the activation, adhesion, crawling, and egress of neutrophils across the endothelial barrier [28]. Chemokines are small cytokines with low molecular mass $(8-10 \mathrm{kDa})$ and are important mediators with chemotactic 
and proactivatory effects on varying leukocyte lineages in inflammation. More than 40 chemokines have been identified so far, and CXCL8 is one of the major chemokines that binds to CXCR1 and CXCR2 receptors to activate intracellular signaling pathways, inducing neutrophils' transfer to the inflammation site, and is associated with the initiation and amplification of acute and chronic inflammatory processes. More than $90 \%$ of the neutrophils' chemotactic activity accumulating in the MSU crystals stimulated site comes from CXCL8 in vitro [21]. The CXCL8 -251T/A (rs4073) polymorphism was reported to be associated with the risk of gout [12].

CXCR1 belongs to the class A rhodopsin-like G-protein coupled receptor (GPCR) and is in charge of cell signaling. The CXCR1 protein encoded gene lies on 2q35 chromosome consisting of two exons separated by an intron [29, 30]. CXCR2 is another GPCR molecule, with similar structure and function triggering chemokine signaling through a tyrosine sulfation motif. The gene that encodes CXCR2 protein also lies on the $2 \mathrm{q} 35$ chromosome and is composed of 3 exons separated by introns [31]. CXCR1 and CXCR2 are broadly expressed in immune cells together, including mast cells, neutrophils, and CD8(+) T cells; neutrophils' recruitment can be prevented by non-competitive allosteric inhibitors of these receptors in vivo, which indicate their pivotal role in mediating neutrophils' transfer to acute inflammation sites [32]. With regard to gout, it was proved that knocking out the murine CXCR-2 homolog (mIL-SRH) can prevent neutrophils from the inflammatory responses to urate crystals in animal models [24].

Our study on the effect of three SNPs in CXCR in the susceptibility of gout in Chinese Han male population showed lack of significant association between genotypes or allele frequencies of rs2234671 or rs1126579 and gout, whereas rs2230054 polymorphism was associated with the development of gout. However, several limitations about this study should not be neglected. First, all the participants were Chinese Han individuals from Shandong Province and could not represent the whole Chinese population, for regional or racial variation having a crucial effect on gout. Second, CXCR1 and CXCR2 genes include numerous variants and only three tag SNPs were selected in the present study, which may give the reason of our failure to find positive outcome in CXCR1. Finally, the sample size of this study was limited. Therefore, further well-designed study with different populations, other polymorphisms, and larger sample sizes are needed to further explore the links between CXCR genetic polymorphisms and the development of gout.

\section{Acknowledgments}

We thank all of the subjects for their participation.

The authors declare no conflict of interest.

\section{References}

1. Miao Z, Li C, Chen Y, et al. (2008): Dietary and lifestyle changes associated with high prevalence of hyperuricemia and gout in the Shandong coastal cities of Eastern China. J Rheumatol 35: 1859-1864.

2. Richette P, Bardin T (2010): Gout. Lancet 375: 318-328.

3. Roddy E, Doherty M (2010): Epidemiology of gout. Arthritis Res Ther 12: 223.

4. Bardin T, Bouée S, Clerson P, et al. (2016): Prevalence of Gout in the Adult Population of France. Arthritis Care Res (Hoboken) 68: 261-266.

5. Riches PL, Wright AF, Ralston SH (2009): Recent insights into the pathogenesis of hyperuricaemia and gout. Hum Mol Genet 18: R177-184.

6. So A, Thorens B (2010): Uric acid transport and disease. J Clin Invest 120: 1791-1799.

7. Merriman TR, Choi HK, Dalbeth N (2014): The Genetic Basis of Gout. Rheum Dis Clin North Am 40: 279-290.

8. Taniguchi A, Kammatani N (2008): Control of renal uric acid excretion and gout. Curr Opin Rheumatol 20: 192-197.

9. Li C, Li Z, Liu S, et al. (2015): Genome-wide association analysis identifies three new risk loci for gout arthritis in Han Chinese. Nat Commun 6: 7041

10. Dehghan A, Kottgen A, Yang Q, et al. (2008): Association of three genetic loci with uric acid concentration and risk of gout: a genome-wide association study. Lancet 372: 19531961.

11. Matsuo H, Yamamoto K, Nakaoka H, et al. (2016): Genome-wide association study of clinically defined gout identifies multiple risk loci and its association with clinical subtypes. Ann Rheum Dis 75: 652-659.

12. Liu S, Yin C, Chu N, et al. (2013): IL-8 -251T/A and IL-12B $1188 \mathrm{~A} / \mathrm{C}$ polymorphisms are associated with gout in a Chinese male population. Scand J Rheumatol 42: 150-158.

13. Landis RC, Haskard DO (2001): Pathogenesis of crystalinduced inflammation. Curr Rheumatol Rep 3: 36-41.

14. Shi Y, Evans JE, Rock KL (2003): Molecular identification of a danger signal that alerts the immune system to dying cells. Nature 425: 516-521.

15. Ghaemi-Oskouie F, Shi Y (2011): The Role of Uric Acid as an Endogenous Danger Signal in Immunity and Inflammation. Curr Rheumatol Rep 13: 160-166.

16. Malawista SE, de Boisfleury AC, Naccache PH (2011): Inflammatory gout: observations over a half-century. FASEB J 25: 4073-4078.

17. Popa-Nita O, Naccache PH (2010): Crystal-induced neutrophil activation. Immunol Cell Biol 88: 32-40.

18. Kelvin DJ, Michiel DF, Johnston JA, et al. (1993): Cheniokines and serpentines: the molecular biology of chemokine receptors. J Leukoc Biol 54: 604-612.

19. Hoch RC, Schraubtaiter IU, Cochrane CG (1996): In vivo, in vitro, and molecular aspects of interleukin- 8 and the interleukin-8 receptors. J Lab Clin Med 128: 134-145.

20. Matsukawa A, Yoshimura T, Maeda T, et al. (1995): Neutrophil accumulation and activation by homologous IL-8 in rabbits. IL-8 induces destruction of cartilage and production of IL-1 and IL-1 receptor antagonist in vivo. J Immunol 154: 5418-5425.

21. Terkeltaub R, Zachariae C, Santoro D, et al. (1991): Monocytic-derived neutrophil chemotactic factor/interleukin- 8 is a pontential mediator of crystal-induced inflammation. Arthritis Rheum 34: 894-903. 
22. Morris SW, Nelson N, Valentine MB, et al. (1992): Assignment of the genes encoding human interleukin- 8 receptor types 1 and 2 and an interleukin- 8 receptor pseudogene to chromosome 2q35. Genomics 14: 685-691.

23. Santos LL, Fan H, Hall P, et al. (2011): Macrophage migration inhibitory factor regulates neutrophil chemotactic responses in inflammatory arthritis in mice. Arthritis Rheum 63: 960-970.

24. Terkeltaub R, Baird S, Sears P, et al. (1998): The murine homolog of the interleukin-8 receptor CXCR-2 is essential for the occurrence of neutrophilic inflammation in the air pouch model of acute urate crystal-induced gouty synovitis. Arthritis Rheum 41: 900-909.

25. Wallace SL, Robinson H, Masi AT, et al. (1977): Preliminary criteria for the classification of the acute arthritis of primary gout. Arthritis Rheum 20: 895-900.

26. Navratilova Z, Gallo J, Mrazek F, Petrek M (2012): Genetic variation in key molecules of the Th-17 immune response is not associated with risk for prosthetic joint infection in a Czech population. Biomed Pap Med Fac Univ Palacky Olomouc Czech Repub 156: 248-252.

27. Hsing AW, Sakoda LC, Rashid A, et al. (2008): Variants in inflammation genes and the risk of biliary tract cancers and stones: a population-based study in China. Cancer Res 68: 6442-6452.

28. Sanz MJ, Kubes P (2012): Neutrophil-active chemokines in vivo imaging of neutrophil trafficking. Eur J Immunol 42: 278-283.

29. Lloyd A, Modi W, Sprenger H, et al. (1993): Assignment of genes for interleukin-8 receptors (IL8R) A and B to human chromosome band 2q35. Cytogenet Cell Genet 63: 238-240.

30. Sprenger H, Lloyd AR, Meyer RG, et al. (1994): Genomic structure, characterization, and identification of the promoter of the human IL-8 receptor A gene. J Immunol 153: 25242532.

31. Sprenger H, Lloyd AR, Lautens LL, et al. (1994): Structure,genomic organization, and expression of the human interleukin-8 receptor B gene. J Biol Chem 269: 11065-11072.

32. Chapman RW, Phillips JE, Hipkin RW, et al. (2009): CXCR2 antagonists for the treatment of pulmonary disease. Pharmacol Ther 121: 55-68. 